\title{
Hints from the Crowd: a Novel NoSQL Database
}

\author{
Paolo Fosci ${ }^{1}$, Giuseppe Psaila ${ }^{1}$, Marcello Di Stefano ${ }^{2}$ \\ 1 University of Bergamo, Dept. of Engineering, \\ Viale Marconi 5, I-24044 Dalmine (BG), Italy, \\ e-mail: paolo.fosci, psaila@unibg.it \\ 2 University of Palermo, Dept. of Computer Science Engineering, \\ Viale delle Scienze Ed. 6, I-90100 Palermo, Italy, \\ e-mail: marcello.distefano@unipa.it
}

\begin{abstract}
The crowd can be an incredible source of information. In particular, this is true for reviews about products of any kind, freely provided by customers through specialized web sites. In other words, they are social knowledge, that can be exploited by other customers.

The Hints From the Crowd (HFC) prototype, presented in this paper, is a NoSQL database system for large collections of product reviews; the database is queried by expressing a natural language sentence; the result is a list of products ranked based on the relevance of reviews w.r.t. the natural language sentence. The best ranked products in the result list can be seen as the best hints for the user based on crowd opinions (the reviews).

In this paper, we mainly describe the query engine, and we show that our prototype obtains good performance in terms of execution time, demonstrating that our approach is feasible. The IMDb dataset, that includes more than 2 million reviews for more than 100,000 movies, is used to evaluate performance.
\end{abstract}

\section{Introduction}

Reviews about products that customers can freely write on specialized web sites constitute an incredible source of information, by means of which users would like to get useful hints. But how could a user obtain them? Typically, the user has some wishes and would like to find products that match those wishes, based on opinions of other users. But to do that, a specialized system is necessary.

Looking at the problem by a database technology point of view, product reviews constitute a text database that has a given structure; user's wishes can be seen as natural language queries over the set of reviews and the user wants to obtain the products whose set of reviews matches the query at the highest degree; the ideal solution, is to get a ranked list, where the best ranked products can be seen as the best hints for the user based on crowd opinions (the reviews). In other words, such a system is a NoSQL database system, where queries are natural language sentences. 
Thus, the Hints From the Crowd (HFC) prototype is a NoSQL database system for large collections of product reviews; the database is queried by expressing a natural language sentence; the result is a list of products ranked based on the relevance of reviews w.r.t. the natural language sentence. Semantic tagging and term expansion (by means of WordNet) are performed, both indexing reviews and querying them.

We wanted to demonstrate that it is possible to obtain an answer to a query in acceptable time on a large set of reviews. Therefore, we tested the prototype on reviews about movies downloaded from the IMDb.com web site, that includes more than 2 million reviews for more than 100,000 movies. The study about execution times at query time is presented.

The paper is organized as follows. Section 2 presents related literature. Section 3 shows and describes the architecture of the system. Section 4 describes how the query engine works and the ranking measure. Section 5 presents the results of the performance study we conducted by means of the IMDb dataset. Finally, Section 6 draws the conclusions.

\section{Related Work}

Research in the database area is more and more addressing the concept of NoSQL database. Several attempts to define the concept can be found. Referring to [14], three categories of data stores are considered: Key-Value stores, Document stores and Column Family stores. The first category deals with datasets similar to maps or dictionaries where data are addressed by a unique key. The second category deals with sets of text documents, and our work falls into this category. The third category encompasses column oriented stores, extensible record stores and wide columnar stores. Graph databases can be considered as well belonging to the world of NoSQL databases [12].

One important aspect of NoSQL data stores is performance. Often, NoSQL databases are necessary due to the implicit limitation of relational databases in given application contexts, where the relational structure of data is an obstacle to obtain fast execution times. The work in [1] tries to address this perspective and presents six features of NoSQL data stores. The are: (1) the ability to horizontally scale simple operation throughput over many servers; (2) the ability to replicate and to distribute (partition) data over many servers; (3) a simple call level interface or protocol (in contrast to a SQL binding); (4) a weaker concurrency model than the ACID transactions of most relational (SQL) database systems; (5) efficient use of distributed indexes and RAM for data storage; (6) the ability to dynamically add new attributes to data records.

The ranking metrics we defined as the basis of the HFC system is inspired to the concept of itemset, developed in the area of data mining for mining frequent itemsets and association rules. Several works $[6,9,7]$ adopt association rule mining for analyzing customer reviews and extract opinions from them. In [6], association rule mining is used to extract, from within customer reviews, 
relevant features the characterize opinions of users about products. In [9], a system to compare opinions about products is presented, where product reviews reports PROs and CONs; in particular, association rule mining is exploited to assign a positive or negative polarity to words (namely, adjectives) in product reviews, and use this polarity to rank the opinion about products. The work in [5] extracts, by means of an association rule mining technique, relevant features that summarize product reviews.

Other data mining techniques are used to analyze customer reviews. For instance, in [10] a sentence clustering technique is adopted. In [2], both supervised and unsupervised approaches are evaluated. A similar work is done in [8], where key classification techniques for opinion mining are discussed. The work in [3] presents a technique for semantic classifications of product reviews.

However, we do not propose a data mining technique and the HFC system is not a data mining system. We simply take inspiration from the concept of frequent itemset.

Proposals related with the notion of frequent itemset and association rule mining was exploited in the area of recommender systems [13]. In effect, the reader could imagine the HFC system as a kind of recommender system. However, based on the general perception about recommender systems (well presented in [11]) a recommender system builds a profile of the user and matches this profile against previous knowledge. In contrast, the HFC system does not build a profile of users; it performs a query, consciously submitted by the user, on the set of product reviews.

Furthermore, notice that certainly the our proposal has some common aspects with search engines and information retrieval techniques. However, these techniques usually models documents based on the Vector Space Model, and do not consider at all the specific features of collections of product reviews.

\section{The HFC System}

As stated in Section 1, Hints From the Crowd is a NoSQL database system that deals with collections of product reviews, that can be queried by expressing a natural language sentence (i.e. query in the rest of the paper).

Due to lack of space, in this Section we shortly mention system architecture and data structure underlying the Query Engine, that we describe in Section 4.

\subsection{Architecture}

The HFC system is composed by several components, each one devoted to perform a specific task as shown in Figure 1. In particular, we distinguish between the back-end and the front-end: the former is responsible for collecting, analyzing and indexing data from product reviews; the latter is the actual user interface accessed by users, that is built on top of the Query Engine.

Let us describe the architecture in details. 


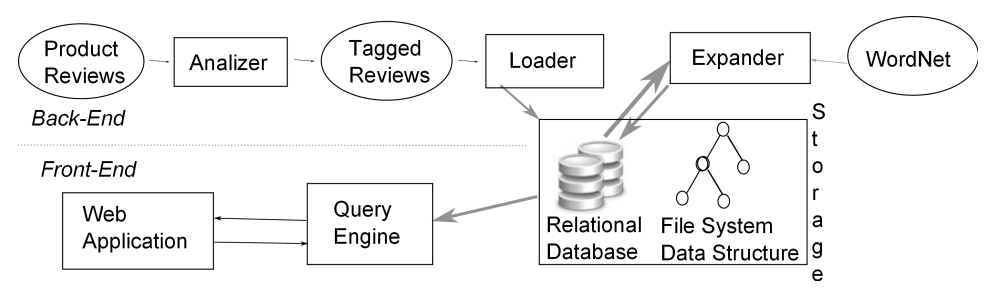

Fig. 1. Architecture of the HFC System.

Back-end. In this side of the system, we find the components (rectangles) that prepares the data structure on which queries are executed. These components operate on source data and intermediate results (ovals) and upload data structures in the Storage box.

- Analyzer. This component is responsible for analyzing product reviews, identifying words and their grammar category (noun, verb, adjective or adverb). This pos-tagging operation is performed by the Stanford Parser $^{3}$. As a result, reviews are transformed into tagged sentences, composed of tagged terms, i.e., a term associated to a tag that denotes the grammar category.

- Loader. The goal of this component is to load Tagged Reviews into the data structures on which queries are performed (see Section 3.2).

- Expander. After the loading phase has ended, tagged terms are expanded on the base of an ontology (in our case, WordNet), so that the Query Engine can exploit semantic relationships in order to capture a wider set of results related with the query.

Front-end. From the architectural point of view, the key component of the frontend is the Query Engine: it exploits the preliminary work performed by back-end components, and works on data structures describing occurrences of terms in product reviews. The Web Application component has been developed to give end users the capability to exploit the system.

\subsection{Data Structure}

Figure 2 graphically depicts the logical schema of the HFC data-structure.

Table Products describes each single product, and its schema is contextdependent, in the sense that the attributes are defined based on the application domain. For example, since we use IMDb movie data set to test the prototype, we defined attributes concerning movies, such as title, director, year, and so on.

Table Terms is the key table, that describes each single tagged word managed by the system. Attributes products, reviews and occurrences counts the number of products and the number of reviews in which a tagged term occurs, and the total number of occurrences, respectively. Notice that, while attribute taggedword denotes a unique element in the table, since a simple word can be associated to more than one grammar category (i.e. word book can be either a noun or a verb), it can appear several times in the table.

\footnotetext{
${ }^{3}$ from the Stanford NLP Group: http://nlp.stanford.edu/index.shtml
} 
Terms(id, word, tag, taggedword, products, reviews, occurrences)

Term2Expansion(termId, expandedWordId, relation)

Occurrences(id, productId, termId, review, position)

Product(id, domain specific attributes)

Fig. 2. Schema of the Relational Database.

Table Term2Expansion represent the relations of a tagged term (by means of attribute termId) with another tagged term (by means attribute expandedTermId). Attribute relation denotes the typology of expansion ${ }^{4}$, i.e., synonym, hypernym, heponym to name a few. Notice that tagging a term with its grammar category (for instance noun), allows us to delimit word expansion only to the relations implied by the grammar category (i.e. the concept of meronym can be applied to a verb, but not to a noun).

Finally, table Occurrences describes all occurrences of tagged terms in product reviews; in particular, notice attribute position, that indicates the position of the occurrence in the review.

Data storing. Due to performance issues, part of the data resides as traditional tables on a relational database (tables Product, Terms and Term2Expansion), and part on the file system (tables Occurrences)

Specifically, as table 0ccurrences is likely to be huge ${ }^{5}$, it has been split in single occurrences file for each term. Each file, containing occurrences of a single term, is identified by the term id. due to the very large number of terms, files are distribuited in a subdirectory tree to avoid to saturate file system limits of files per directory.

Internally, each occurrences file is organized as a binary file, where a fixed length data structure represents a term occurrence; this data structure is a 12 bytes triple (ProductId, ReviewId, Position).

Furthermore, for the sake of performance study, we also have a 2nd version of the file system data structure, where occurrences are partitioned in 5 orthogonal subtrees, and each subtree describes occurrences for $1 / 5$ of the products. This second version allows us to implement a multi-thread query engine, with 5 threads running in a parallel way (see 5.2).

\section{Query Engine}

We now describe the key component, i.e., the query engine. Based on a natural language sentence (the query) it extracts those products whose reviews are mostly relevant for the query. Relevance is evaluated by means of a ranking metric; retrieved products are returned as a list sorted in reverse order of relevance. Hereafter, we describe how the ranking metric is defined.

\footnotetext{
${ }^{4}$ WordNet provides a set of 15 different possible expanding relations depending on word grammar category

${ }^{5}$ In our test case, the size is more than $12 \mathrm{~Gb}$
} 


\begin{tabular}{|c|c|c|}
\hline$l \# 1$ & weight & termsets (I) \\
\hline 41 & 0.5000 & $\{$ funny, great, hilarious, jokes $\}$ \\
\hline 34 & 0.1000 & $\begin{array}{l}\text { \{funny, great, hilarious } \\
\text { \{funny, great, jokes } \\
\text { funny, hilarious, jokes } \\
\text { \{great, hilarious, jokes }\end{array}$ \\
\hline 26 & 0.0167 & $\begin{array}{l}\{\text { funny, great }\{\text { funny, hilarious } \\
\text { \{great, hilarious }\} \text { \{great, jokes } \\
\text { \{funny, jokes }\{\text { hilarious, jokes }\end{array}$ \\
\hline
\end{tabular}

Fig. 3. Termsets levels for query great funny hilarious jokes and corresponding weights.

\subsection{Termsets}

In this paper we consider a query $q$ as a set of terms (or briefly, a termset). Thus, we describe a query containing a number $n$ of terms as $q=\left\{t_{1}, \ldots, t_{n}\right\}^{6}$, and we investigate only those queries where $n>1$ or, in other words, $|q|>1$.

With $I$, we denote a generic termset that is a subset of $q$ for which applies $|I|>1$. With $D_{q}$, we denote the set of termsets $I$ derived from $q$. Notice that the cardinality of $D_{q}$ is $\left|D_{q}\right|=2^{n}-(n+1)$, i.e. $D_{q}$ is the power set of $q$ without the empty set and the $n$ single terms that compose $q$.

With $I_{l}$ we denote an $l$-termset of $q$, that is a termset composed by $l$ terms, i.e. $\left|I_{l}\right|=l$. With $D_{q, l}$ we denote the set of $l$-termsets $I_{l}$. Notice that the cardinality of $D_{g, l}$ is $\left|D_{q, l}\right|=\left(\begin{array}{l}n \\ l\end{array}\right)$.

\subsection{Termset Weight}

We now define the concept of weight for a termset.

Definition 1: The weight of a $l$-termset is a function of its length and the length of the query $q(|q|=n)$ and it is denoted as $w_{q}(l)$.

For $n=2$ there is only one 2 -termset and its weight is $w_{q}(2)=1$ by definition.

For $n>2$ the weight of the single $n$-termset $q$ is, by definition, $w_{q}(n)=0.5$, while for $2<l<n$ it is $w_{q}(l)=w_{q}(l+1) /\left(\left(\begin{array}{l}n \\ l\end{array}\right)+1\right)$ and for $l=2$ it is $w_{q}(2)=w_{q}(3) /\left(\begin{array}{c}n \\ 2\end{array}\right)$.

The rationale behind Definition 1 is the following. The topmost termset, corresponding to the whole query, is the most important one, and its weight is equal to the overall weight of all the shorter termsets. The same principle is valid for any generic termset $I_{l}$ (with $2<l<n$ ), whose weight is equal to the overall weight of all lower levels termsets (even those that are not subset of $I_{l}$ ). In this way, reducing the size of termsets, the contribution of each level quickly decreases.

Notice, that the overall weight of all termsets is exactly $1\left(\sum_{I \in D_{q}} w_{q}(|I|)=1\right)$.

Figure 3 shows the termsets levels with an example query.

\subsection{Query Expansion and Semantic Coefficient}

As stated in Section 3.1, reviews are processed performing several operations. Similar operations are performed on a user query in natural language as well.

\footnotetext{
${ }^{6}$ At moment, in this stage of the project we do not consider word order or repetitions
} 
Pos-tagging. By means of Stanford Parser, each word of a user query is tagged with an attribute that denotes its grammar role (verb, noun, adjective to name a few) in the query.

Stopwords filtering. Stopwords are those words that are too common in reviews (such as articles, conjunctions); furthermore, common verbal forms like is or have (just to name a few) are treated as stopwords. Stopwords include also some very context-dependent words such as the word actor in a movie context. These words hold a small semantic meaning, so after pos-tagging operation stopwords are discarded from the query.

Thus, denoting with $S W$ the set of possible stopwords, in the rest of the paper the notation:

$$
q=\left\{t_{1}, \ldots, t_{n}\right\}
$$

includes only those terms $t_{i} \notin S W$, and, as stated in Section 4.1, we consider only those queries $q$ such that $|q|>1$ (actual length without stopwords).

Term expansion. By means of WordNet ontology, each tagged term $t_{i} \in q$ is expanded with all those terms directly associated to $t_{i}$ depending by its grammar tag. Thus, for example a noun is expanded with all its synonyms, hypernyms or hyponyms and so on, while a verb is expanded with all its synonyms or meronyms, to name a few. There are actually a total of 15 possible different relations between a tagged term and its expanded words.

We denote with $t_{i}^{*}$ the generic expanded term of $t_{i}$, and with $E T\left(t_{i}\right)$ the set of all expanded terms of $t_{i}$. By definition, $t_{i} \in E T\left(t_{i}\right)$ with an identity relation, thus, $\left|E T\left(t_{i}\right)\right| \geq 1$.

Notice that, given a generic expanded term $t^{*}$, it can happen that $t^{*} \in E T\left(t_{i}\right)$ and $t^{*} \in E T\left(t_{j}\right)$ with $i \neq j$. In other words, we cannot state a-priori that $E T\left(t_{i}\right) \cap E T\left(t_{j}\right)=\oslash$ with $i \neq j$. As an example, the term colour can be an hypernym expansion for both terms red and black.

Query expansion. An expanded query $q^{*}$ is each combination of $\left\{t_{1}^{*}, \ldots, t_{n}^{*}\right\}$. We consider valid a combination $q^{*}=\left\{t_{1}^{*}, \ldots, t_{n}^{*}\right\}$ only if $t_{i}^{*} \neq t_{j}^{*} \forall i \neq j$ Notice that the original query $q$ is a particular $q^{*}$ itself, and it is valid by definition.

Expanded termsets. Previous considerations about query $q$ and its expansions, are applicable to each termset $I_{l}$. With $I_{l}^{*}$ we denote an expanded termset $I_{l}^{*}=$ $\left\{t_{1}^{*}, \ldots, t_{l}^{*}\right\}$, and similarly $I_{l}^{*}$ is valid only if $t_{i}^{*} \neq t_{j}^{*} \forall i \neq j$.

With $E I(I)$ we denote the set of all possible expanded termset $I^{*}$ that can be derived from $I$. The cardinality of $E I(I)=\prod_{t \in I}|E T(t)|$, that is the number of all possible combinations of the expanded terms of those terms that compose $I$.

Finally, with $D_{q}^{*}$, we denote the set of all valid expanded termsets that are included in $q$ and all its valid expansions $q^{*}$. 


\begin{tabular}{r|c|c}
\hline$|E T(t)|$ & $s c_{t}(t)$ (not expanded) & $s c_{t}\left(t^{*}\right)\left(t^{*} \neq t\right)$ \\
\hline 1 & 1.0000 & - \\
2 & 0.7500 & 0.2500 \\
3 & 0.6667 & 0.1667 \\
5 & 0.6000 & 0.1000 \\
10 & 0.5500 & 0.0500 \\
\hline \multicolumn{2}{c}{ a) }
\end{tabular}

\begin{tabular}{c|c|c}
\hline$I^{*}$ (structure) & $\#$ & $s c_{t}\left(I^{*}\right)$ \\
\hline$\left\{t_{1}, t_{2}\right\}$ & 1 & 0.4444 \\
$\left\{t_{1}^{*}, t_{2}\right\}$ or $\left\{t_{1}, t_{2}^{*}\right\}$ & 4 & 0.1111 \\
$\left\{t_{1}^{*}, t_{2}^{*}\right\}$ & 4 & 0.0278 \\
\hline
\end{tabular}

b)

Table 1. a) Trend of Semantic coefficient w.r.t. the cardinality of $E T(t)$. b) Semantic coefficient for a generic 2-termset where each term has two expansions.

Semantic coefficient. Each $t^{*} \in E T(t)$ has a semantic coefficient $s c_{t}\left(t^{*}\right)$, with $0<s c_{t}\left(t^{*}\right) \leq 1$, that depends on the cardinality of $E T\left(t_{i}\right)$.

Definition 2: For each $t^{*} \in E T(t)$ except $t, s c_{t}\left(t^{*}\right)=0.5 /|E T(t)|$, and $s c_{t}(t)=$ $0.5+0.5 /|E T(t)|$.

The rationale of semantic coefficient, is the following. A term describes a semantic concept that is mostly expressed by the term itself, but receives a small contribution from expanded terms: the greater the number of expansion, the smaller the semantic contribution of a single expanded term. Notice that $\sum_{t^{*} \in E T(t)} s c_{t}\left(t^{*}\right)=1$. Table 1.a shows how $s c_{t}$ varies with $E T(t)$ cardinality.

With $s c_{I}\left(I^{*}\right)$ we denote the semantic coefficient for an expanded termset $I^{*}$ derived from $I$.

Definition 3: Given an expanded termset $I^{*}=\left\{t_{1}^{*} \ldots t_{l}^{*}\right\}$ derived for a termset $I=\left\{t_{1} \ldots t_{l}\right\}$, it is $s c_{I}\left(I^{*}\right)=\prod_{t_{i}^{*} \in I_{l}^{*}} s c_{t_{i}}\left(t_{i}^{*}\right)$.

This way, a termset that contains only original terms gives the highest semantic contribution, while augmenting the number of expanded terms in the termset, the semantic contribution decreases.

Table 1.b shows the trend of the semantic coefficient for a 2-termset where each original term has two expansions. Notice that, according to the above definition, $\sum_{I^{*} \in E I(I)} s c_{I}\left(I^{*}\right)=1$.

\subsection{Product Reviews and Termsets}

Consider a product $p$ (a movie, a camera, etc.), its set of reviews is denoted by $R(p)=\left\{r_{1}, \ldots, r_{k}\right\}$. Each review is a text, i.e., a sequence of term occurrences $r_{i}=<t_{1}, \ldots, t_{s}>$.

With $T(R(p))$ we denote the set of terms appearing in reviews for product $p$, and with $T\left(r_{i}\right)$ the set of terms appearing in review $r_{i} \in R(p)$.

Definition 4: A termset $I$ is said relevant for product $p$ if $\exists r_{i} \mid I \subseteq T\left(r_{i}\right)$.

The set of relevant termsets for product $p$ is denoted as $R D_{p, q}$. In an analogous way, $R D_{p, q}^{*}$ is the set of all relevant expanded termsets for product $p$. Notice that $R D_{p, q} \subseteq D_{q}$, and also $R D_{p, q}^{*} \subseteq D_{q}^{*}$.

\subsection{Termset Average Density}

In a preliminary work [4], we assumed that every termset occurrence in product reviews contribute to the support of the termset with the same weight, i.e. 1 , 
since the support, by definition, is the number of reviews containing the termset on the total amount of reviews.

Given a termset $I$, in a single review, terms in $I$ can be very dense or, on the opposite case, very sparse. We consider a review in which the occurrences of terms in $I$ are dense being more relevant for the query than a review where occurrences are sparse. Thus, we introduce the concept of Termset Density of an termset $I$ for a single review.

Definition 5: Consider a product $p$, a review $r \in R(p)$, and a termset $I_{l}$. The Termset Review Density $d_{r}\left(I_{l}\right)$ is defined as

$$
d_{r}\left(I_{l}\right)=l / \min \operatorname{Win}_{r}\left(I_{l}\right)
$$

where $\min \operatorname{Win}_{r}\left(I_{l}\right)$ is the size of the minimal window in review $r$ that includes all the terms of termset $I_{l}$.

Notice that for Termset Review Density, it holds that $0<d\left(I_{l}, r\right) \leq 1$

The next step is to define a Termset Average Density for a generic termset $I$ (we omit the subscript $l$ not to burden notation) w.r.t. a product $p$.

Definition 6: Consider a product $p$ and its set of reviews $R(p)$. With $R_{I}(p)$ we call the subset of $R(p)$ of those reviews containing termset $I$. The Termset Average Density for product $p$, denoted as $a d_{p}(I)$, is defined as:

$$
a d_{p}(I)=\left(\sum_{r \in R_{I}(p)} d_{r}(I)\right) /|R(p)|
$$

The Termset Average Density is analogous to termset support, with the difference that the contribution of the occurrence of a termset $I$ in a review $r$ is not 1 but its density $d_{r}(I)$. Notice, thus, that $a d_{p}(I) \leq s_{p}(I) \leq 1$, where with $s_{p}(I)$ we denote the support of a termset $I$ for a product $p$.

\subsection{Product Ranking Metric}

Finally, we can now define the Product Ranking Metric PRM.

Definition 7: Consider a query $q$, the set of termsets $D_{q}^{*}$ derived from $q$, the system of the weights $w_{q}\left(\left|I^{*}\right|\right)$ and semantic coefficients $s c_{q}\left(I^{*}\right)$ for each expanded termset $I^{*} \in D_{q}^{*}$.

Consider a product $p$, the set of reviews $R(p)$ and the set of relevant expanded termsets $R D_{p, q}^{*}$ that can be actually extracted from $R(p)$. Given for each $I^{*} \in R D_{p, q}^{*}$ the average termset density $a d_{p}\left(I^{*}\right)$, the Product Relevance Value for product $p$ is defined as

$$
P R M_{q}(p)=\sum_{I^{*} \in R D_{p, q}^{*}}\left(w_{q}\left(\left|I^{*}\right|\right) \times a d_{p}\left(I^{*}\right) \times s c_{q}\left(I^{*}\right)\right)
$$

The rationale of the above definition is the following. For each termset $I^{*}$ included in the query $q$ and actually relevant in the reviews, its contribution to the overall relevance value is given by its weight $w_{q}\left(\left|I^{*}\right|\right.$ ) (that depends on its size) multiplied by its average density $a d_{p, q}\left(I^{*}\right)$ and its semantic coefficient $s c_{q}\left(I^{*}\right)$.

The system of weights and semantic coefficients has been designed to obtain a $\operatorname{PRM}_{q}(p)=1$ for an ideal set of reviews for product $p$, where each review 


\begin{tabular}{lrrr}
\hline Schema & $\mathrm{A}$ & $\mathrm{B}$ & Diff \% \\
\hline Pos-Tagger & active & inactive & \\
Distinct tagged terms & $1,151,827$ & 776,852 & $-32.55 \%$ \\
Occurrences & $216,345,522$ & $216,345,522$ & $0.00 \%$ \\
\hline Analysis Time (A = Ps+Pt) & $2226.80 \mathrm{~h}$ & $3.82 \mathrm{~h}$ & $-99.83 \%$ \\
$\quad$ Parsing Time (Ps) & $2.11 \mathrm{~h}$ & $2.42 \mathrm{~h}$ & $+14.74 \%$ \\
$\quad$ Pos-tagging Time (Pt) & $2224.69 \mathrm{~h}$ & $1.40 \mathrm{~h}$ & $-99.94 \%$ \\
Db Loading Time (D) & $56.05 \mathrm{~h}$ & $49.76 \mathrm{~h}$ & $-11.23 \%$ \\
Term Expansion Time (E) & $3.73 \mathrm{~h}$ & $2.67 \mathrm{~h}$ & $-28.49 \%$ \\
Total Time (T = A+D+E) & $2286.58 \mathrm{~h}$ & $56.25 \mathrm{~h}$ & $-97.54 \%$ \\
\hline \multicolumn{4}{c}{ Table 2. Indexed schemes } \\
\hline
\end{tabular}

contains every expanded termset $I^{*}$ that can be derived from $q$ with a density $d_{r}\left(I^{*}\right)=1$, and every expanded termset $I^{*}$ is valid.

\section{Evaluation}

Our dataset is composed by a total of 2,207,678 user reviews for 109,221 movies downloaded from the IMDb.com web site. The size of the text we downloaded is approximatively 3,091 Mb. Each movie has a number of reviews included between 1 and 4,876, and the average number of reviews per movie is 20.

Experiments has been run on a PC with two Intel Xeon Quad-core 2.0GHz/L3$4 \mathrm{MB}$ processors, 12GB RAM, four 1-Tbyte disks and Linux operating system.

\section{$5.1 \quad$ Indexing}

While indexing our data set, as described in the back-end side of HFC system architecture in 3.1, we figured out how pos-tagging affects the HFC system.

Disabling pos-tagging means tagging each term with a unique trivial tag, and considering for each term every possible expansion regardless of its role inside the query; in other words, disabling pos-tagging means a significant reduction of the number of managed terms because words are distinguished on the basis of their grammar category (for instance word colour could be both a noun and a verb); however, the counter effect is that the possible number of expansions for a termset combinatorially increases.

Table 2 reports data collected during dataset indexing. Column $A$ shows data regarding indexing with pos-tagging activated (Schema $A$ ), while Column $B$ shows data regarding indexing with pos-tagging deactivated (Schema B). Column Diff \% shows the percentage variation from data of Schema A to data of Schema B (where applicable). For each Schema, the total number of indexed term occurrences (row Occurrences) and the number of identified tagged terms are shown. As said in the premises, disabling pos-tagging reduces the number of tagged terms $(\sim 33 \%)$. Table 2 reports, in rows, also data relative to execution time (in hours) during the indexing phase. The Total Time of the indexing is given by the sum of Analysis Time, Db Loading Time and Term Expansion Time. Moreover Analysis Time is split in Parsing Time, that is basically the time due to reading data from data set, and Pos-tagging Time, that considers 


\begin{tabular}{lrrr}
\hline & Single-thread & 5-threads & Diff \% \\
\hline Average Time (T=QE+TG+TE+TM+S) & $2,501.12 \mathrm{~ms}$ & $1,994.66 \mathrm{~ms}$ & $-20.25 \%$ \\
\hline Query Expansion (QE & $286.44 \mathrm{~ms}$ & $286.40 \mathrm{~ms}$ & $-0.01 \%$ \\
Thread generation (TG) & $0.40 \mathrm{~ms}$ & $1.88 \mathrm{~ms}$ & $370.00 \%$ \\
Thread execution (TE $\leq \mathrm{O}+\mathrm{R})$ & $2,199.64 \mathrm{~ms}$ & $1,691.60 \mathrm{~ms}$ & $-23.10 \%$ \\
Occurrences Loading (O) & $1,962.52 \mathrm{~ms}$ & $1,639.84 \mathrm{~ms}$ & $-16.44 \%$ \\
Ranking (R) & $237.12 \mathrm{~ms}$ & $75.12 \mathrm{~ms}$ & $-68.32 \%$ \\
Thread merging (TM) & $1.64 \mathrm{~ms}$ & $1.80 \mathrm{~ms}$ & $9.76 \%$ \\
Sorting (S) & $13.00 \mathrm{~ms}$ & $12.98 \mathrm{~ms}$ & $-0.17 \%$ \\
\hline
\end{tabular}

Table 3. Single-thread search engine Vs 5-threads search engine

only the execution time of Stanford Parser when pos-tagging is active, and instead the simple operation of labeling each term with the same tag when the pos-tagging is inactive. It is clearly evident how much the Analysis Time is affected by Pos-tagging Time using Stanford Parser: the more than 2200 hours needed for pos-tagging are equivalent to more than 90 days! In order to reduce this waiting time, we exploited all the 8 cores of the machine used for the experiment, parallelizing the Analysis phase in 8 independent processes, splitting data set into 8 different sub-data sets, and reducing the actual waiting time to about 13 days.

The analysis of variation of Db Loading Time and Term Expansion Time highlights how the higher number of tagged terms in Schema $A$ w.r.t. Schema $B$ affects the execution time of the Loader and the Expander.

\subsection{Query Performance}

For our query performance tests we prepared a set of 25 standard user queries ${ }^{7}$ like I want to know more about the history of Greece and the Persian wars, or All those moments will be lost in time, like tears in rain ${ }^{8}$.

The first test we made, compares the variation of performance of the query engine working on Schema $A$ (ad described in Section 5.1) in a single-searchingthread version versus a 5 -searching-threads version. Table 3 shows the average results of the test performed on the set of 25 standard queries mentioned before. Column Single-thread shows performance of the single-thread search engine, while Column 5-threads shows performance of the 5-threads search engine, and column Diff \% shows the percentage variation from single-thread w.r.t. 5 -threads search engine. For each search engine version, the average execution time per query is provided in row Average Time.

Basically, the query engine evaluates a query performing 4 different steps: (1) query expansion, (2) occurrences loading, (3) product ranking, (4) result sorting. Steps 2 and 3 can be parallelized (and performed in different threads), while Steps 1 and 4 must be performed by a single thread.

Row Thread execution of Table 3 reports the average execution time of the slowest searching threads for each query, while rows Occurrences Loading and Ranking report the average time of the slowest thread in executing respectively Step 2 and Step 3 for each query; that is why $T E \leq O+R$.

\footnotetext{
${ }^{7}$ due to lack of space, we don't report the testing queries

8 from Blade Runner movie
} 


\begin{tabular}{lrrr}
\hline Schema & $\mathrm{A}$ & $\mathrm{B}$ & Diff \% \\
Pos Tagging & active & inactive & \\
\hline Total time & $1,995 \mathrm{~ms}$ & $3,480 \mathrm{~ms}$ & $74.47 \%$ \\
Movies & 2,067 & 2,994 & $44.85 \%$ \\
Occurrences & 107,200 & 226,994 & $111.75 \%$ \\
Termsets & 5,414 & 13,795 & $154.80 \%$ \\
\hline Table 4. Pos tagging VS No-pos tagging
\end{tabular}

The analysis of execution times in Table 3 shows that most of the time needed to perform a query is because of occurrences loading: this is mostly due to our storage system based on classical hard disks. With more modern solid-state storage system, that are at least one order of magnitude faster, we are confident to dramatically improve performance.

Another issue is about threads parallelization. From the compared analysis, at first glance could seems that the 5 -thread search engine version has not significantly improved performance, since there is only a $16.44 \%$ of gain in occurrences loading. However, this is mostly due to the fact that we have de facto a single storage system: data are transferred to main memory through a single system bus. We are confident that parallelized the process on different machines performance should dramatically increased. As a matter of fact, the compared analysis of ranking execution times, that do not involve disk use, tells that 5-treads search engine is $68.32 \%$ more perfoming that the single-thread search engine.

The second test we have made on our set of 25 queries, is a comparison between performance of the 5-thread search engine on the Schema A and Schema $B$ as described in Section 5.1. Table 4 shows in column $A$ data related to Schema $A$, and in column $B$ data related to Schema $B$. Column Diff \% shows the percentage variation from $S$ chema $A$ w.r.t. Schema $B$.

Table 4 provides average data-per-query. It can be noticed that when pos-tagger is inactive there is a growing of average execution time, mostly due to the larger number of occurrences to load, and also to a larger number of termsets to analyze. On the other hands, there is larger number of movies retrieved as, actually, deactivating pos-tagging means increasing the number of expanded terms to search (causing generation of false positive movies, i.e., movies whose reviews are not actually relevant w.r.t. the original query).

\section{Conclusions}

The scope of this paper was to present the architecture and the query engine of $H F C$ NoSQL database system. Although performance of the system can be further be improved, the considerations in 5.2 show that the approach is feasible in terms of query response time.

We are aware we did not discuss about system effectiveness, but it was beyond the scope of the paper. However the web-interface we developed is designed to collect users opinions about the system, and by means of that, in the future work we intend to deeper investigate effectiveness of the system. Moreover, as far as effectiveness is concerned, in the future work we intend to integrate term 
expansion with linked-data as a source for semantic ontology about terms, and also considering word order and word repetition in queries in our ranking model.

\section{References}

1. R. Cattell. Scalable sql and nosql data stores. SIGMOD Record, 39 (4):12-27, 2011.

2. P. Chaovalit and L. Zhou. Movie review mining: a comparison between supervised and unsupervised classification approaches. In HICSS. IEEE Computer Society, 2005.

3. K. Dave, S. Lawrence, and D. M. Pennock. Mining the peanut gallery: opinion extraction and semantic classification of product reviews. In $W W W$, pages 519 $528,2003$.

4. P. Fosci and G. Psaila. Toward a product search engine based on user reviews. In DATA-2012 Int. Conf. on Data Technologies and Applications, Rome (Italy), July 2012.

5. M. Hu and B. Liu. Mining and summarizing customer reviews. In W. Kim, R. Kohavi, J. Gehrke, and W. DuMouchel, editors, KDD, pages 168-177. ACM, 2004.

6. M. Hu and B. Liu. Mining opinion features in customer reviews. In D. L. McGuinness and G. Ferguson, editors, AAAI, pages 755-760. AAAI Press / The MIT Press, 2004.

7. W. Kim, J. Ryu, K. I. Kim, and U.-M. Kim. A method for opinion mining of product reviews using association rules. In S. Sohn, L. Chen, S. Hwang, K. Cho, S. Kawata, K. Um, F. I. S. Ko, K.-D. Kwack, J. H. Lee, G. Kou, K. Nakamura, A. C. M. Fong, and P. C. M. Ma, editors, Int. Conf. Interaction Sciences, volume 403 of ACM International Conference Proceeding Series, pages 270-274. ACM, 2009.

8. D. Lee, O.-R. Jeong, and S. goo Lee. Opinion mining of customer feedback data on the web. In W. Kim and H.-J. Choi, editors, ICUIMC, pages 230-235. ACM, 2008.

9. B. Liu, M. Hu, and J. Cheng. Opinion observer: analyzing and comparing opinions on the web. In A. Ellis and T. Hagino, editors, $W W W$, pages 342-351. ACM, 2005.

10. D. K. Ly, K. Sugiyama, Z. Lin, and M.-Y. Kan. Product review summarization based on facet identification and sentence clustering. CoRR, abs/1110.1428, 2011.

11. P. Resnick and H. Varian. Recommender systems. Communications of the ACM, 40(3):5658, 1997.

12. H. Robin and S. Jablonski. Nosql evaluation: A use case oriented survey. In CSC-2011 International Conference on Cloud and Service Computing, Hong Kong, China, pages 336-341, December 2011.

13. J. J. Sandvig, B. Mobasher, and R. D. Burke. Robustness of collaborative recommendation based on association rule mining. In J. A. Konstan, J. Riedl, and B. Smyth, editors, RecSys, pages 105-112. ACM, 2007.

14. C. Strauch. Nosql databases. http://www.christof-strauch.de/nosqldbs.pdf, 2011. 